\title{
Diabetes per se verringert Funktionsdauer koronarer Bypässe nicht
}

Fragestellung: Ist die aortokoronare Bypass-Operation (coronary artery bypass graft $=$ CABG) bei Diabetikern weniger erfolgreich als bei Nichtdiabetikern?

Hintergrund: Etwa 50 \% der Patienten, die einen aortokoronaren Bypass erhalten, sind Diabetiker. Trotzdem gibt es kaum Daten zum Einfluss der diabetischen Stoffwechsellage auf die Durchgängigkeit und Verschlussrate der Transplantate im postoperativen Verlauf. Die Autoren gingen von der Hypothese aus, dass Stenosierungen und Verschlüsse bei Diabetikern häufiger sind, weil das Risiko für arteriosklerotische Gefäßveränderungen größer ist. Sie überprüften diese Annahme bei venösen und arteriellen Gefäßprothesen (Vena saphena bzw. Arteria thoracica interna).

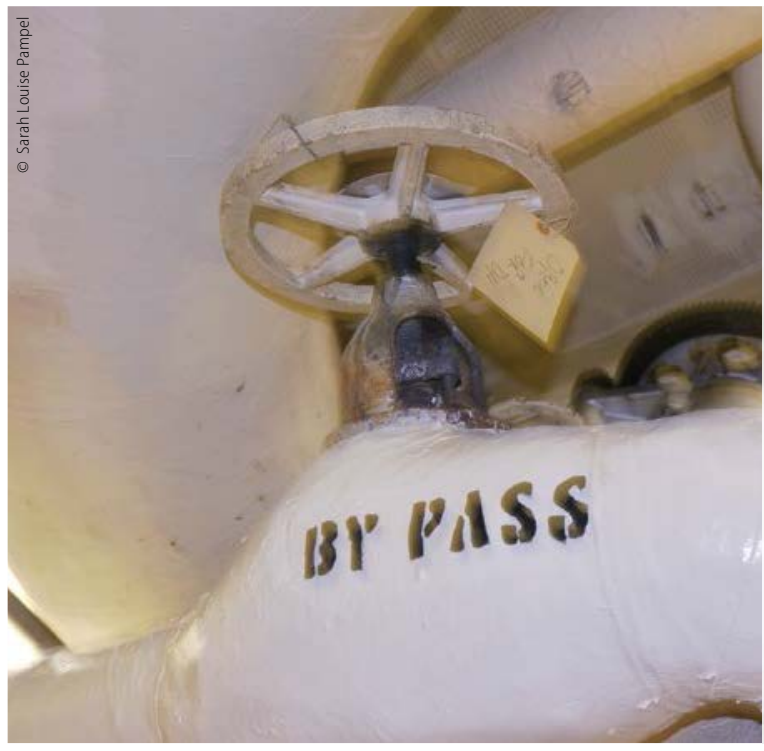

Ob der Hahn am Herzen offen bleibt, hängt maßgeblich vom Ersatzgefäß und der Therapie der kardiovaskulären Risikofaktoren bei Diabetikern $a b$.
Patienten und Methoden: Von 1972 bis 2011 erhielten 57.961 Patienten an der Cleveland-Klinik erstmalig einen aortokoronaren Bypass. Von 11.519 Patienten, davon 1.372 Diabetiker, waren Angiogramme mit dem genauen präoperativen Koronargefäßstatus, mindestens einem postoperativen Angiogramm einschließlich quantitativer Beschreibung der Bypass-Morphologie verfügbar.

Ergebnisse: Die Durchgängigkeit der arteriellen Gefäßprothesen lag konstant sehr hoch und war bei Diabetikern und Nichtdiabetikern nach 1, 5, 10 und 20 Jahren nahezu identisch (97, 97, 96 bzw. 96 \% bei Diabetikern, 96, 96, 95 und $93 \%$ bei Nichtdiabetikern). Anders war es beim Bypass mit venösen Gefäßen: Hier kam es im gleichen Zeitraum zu einem deutlichen Abfall der Durchgängigkeit, aber ebenfalls ohne Unterschied zwischen Diabetikern und Nichtdiabetikern $(78,70$, 57 und $42 \%$ bzw. 82, 72, 58 und $41 \%$ ). Die Überlebensrate 20 Jahre nach CABG betrug bei Diabetikern $35 \%$, bei Nichtdiabetikern $58 \%$.

Schlussfolgerung: Bei Diabetikern und Nichtdiabetikern ist die Durchgängigkeit des arteriellen koronaren Bypasses langfristig deutlich höher als bei einem

\section{Originalie}

Raza S, Blackstone E H, Houghtaling P L et al. Influence of Diabetes on Long-Term CoronaJACC 2017;70:515-24 ry Artery Bypass Graft Patency. venösen Bypass. Andererseits haben Diabetiker und Nichtdiabetiker, die den gleichen, d.h. einen venösen oder einen arteriellen Bypass erhalten, auf lange Sicht die gleiche Durchgängigkeit.

- Kommentar von Prof. Dr. med. H. Holzgreve Nicht der Diabetesstatus, der
Bypasstyp ist entscheidend

Vielfach wird angenommen, dass koronare Gefäßbrücken bei Diabetikern weniger lange halten und diese Operation daher einen geringeren Nutzen hat als bei Nichtdiabetikern. Die vorliegende umfangreiche und langfristige Beobachtungsstudie zeigt nun, dass die Durchgängigkeit von arteriellen Gefäßbrücken 20 Jahre postoperativ über $90 \%$ und bei venösen über $40 \%$ liegt, jeweils ohne Unterschied zwischen Diabetikern und Nichtdiabetikern. Es mutet paradox an, wenn gleichzeitig die Überlebensrate nach CABG bei Diabetikern niedriger liegt als bei Nichtdiabetikern. Die schlechte Prognose der Diabetiker beruht demnach nicht auf Mängel der Bypass-Operation, sondern auf Komorbiditäten, progressiver Arteriosklerose und Komplikationen des Diabetes. Nach dem Chirurgen muss also der Diabetologe ran, um die Prognose zu verbessern!

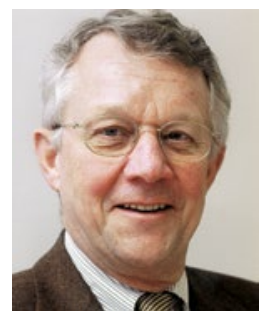

Prof. Dr. med. Heinrich Holzgreve

Internist, Kardiologische Praxis Burgstr. 7, 80331 München

heinrich.holzgreve@t-online.de 